\title{
Controlling and characterising the deposits from polymer droplets containing microparticles and salt ${ }^{\star}$
}

\author{
Y. Msambwa, A.S.D. Shackleford, F.F. Ouali, and D.J. Fairhurst ${ }^{\mathrm{a}}$ \\ Nottingham Trent University, Clifton Lane, Nottingham, NG11 8NS, UK
}

Received 14 July 2015 and Received in final form 29 January 2016

Published online: 26 February 2016

(c) The Author(s) 2016. This article is published with open access at Springerlink.com

\begin{abstract}
A coffee ring-stain is left behind when droplets containing a wide range of different suspended particles evaporate, caused by a pinned contact line generating a strong outwards capillary flow. Conversely, in the very peculiar case of evaporating droplets of poly(ethylene oxide) solutions, tall pillars are deposited in the centre of the droplet following a boot-strapping process in which the contact line recedes quickly, driven by a constricting collar of polymer crystallisation: no other polymer has been reported to produce these central pillars. Here we map out the phase behaviour seen when the specific pillar-forming polymer is combined with spherical microparticles, illustrating a range of final deposit shapes, including the standard particle ring-stain, polymer pillars and also flat deposits. The topologies of the deposits are measured using profile images and stylus profilometery, and characterised using the skewness of the profile as a simple analytic method for quantifying the shapes: pillars produce positive skew, flat deposits have zero skew and ring-stains have a negative value. We also demonstrate that pillar formation is even more effectively disrupted using potassium sulphate salt solutions, which change the water from a good solvent to a thetapoint solvent, consequently reducing the size and configuration of the polymer coils. This inhibits polymer crystallisation, interfering with the bootstrap process and ultimately prevents pillars from forming. Again, the deposit shapes are quantified using the skew parameter.
\end{abstract}

\section{Introduction}

The work of Deegan et al. [1] first thoroughly investigated the properties of the coffee ring-stain, commonly seen when suspension droplets are left to evaporate on a solid surface. They proposed a simple explanation for these deposits with just two requirements: firstly, the triple line at the edge of the droplet must remain pinned to the substrate throughout (nearly all of) the drying process, known as constant contact radius drying (CCR) [2]; secondly the evaporative flux over the droplet varies with radius $r$ measured from the centre of the droplet and diverges at the contact line $r=R$ following a power law. These two requirements lead to an outward flow to replenish solvent loss at the contact line, which sweeps suspended material to the contact line where it is deposited as a ring-stain. The size and shape of the deposit is robust over a range of experimental parameters and follows simple power-law predictions [3]. One of the aims of ongoing research into drying sessile droplets is to control and

\footnotetext{
* Contribution to the Topical Issue "Wetting and Drying. Physics and Pattern Formation", edited by Duyang Zang, Ludovic Pauchard and Wei Shen.

a e-mail: david.fairhurst@ntu.ac.uk
}

prevent the formation of the coffee ring-stain as many commercial processes require a uniform deposit. Several mechanisms have been observed to achieve this goal including: non-spherical particles [4], capillary forces [5]; Marangoni flow induced by surface tension gradients [6]; electrowetting [7]; using droplets smaller than a critical size [8] and heated substrates [9]. Many of these effects are summarised in a recent review [10].

In many cases, ring-stains are also suppressed if the liquid in the droplet undergoes a phase-change during evaporation. For example, in drying droplets of both dextran [11] and bitumen [12] the contact line becomes pinned, a flexible glassy skin with fixed surface area forms and as evaporation continues the film buckles leaving a final deposit in the shape of a sombrero.

The polymer used in this study is the very widely used linear polymer PEO, poly(ethylene oxide) [13-16]. It is unique amongst its homologues for its unusual solubility properties [17]: it dissolves in water, although at high concentrations or molecular weights, solutions can appear cloudy due to micron-sized clusters of undissolved polymer [18]. The origin of these clusters is still a point of contention [18]. The properties of PEO are very well known including data on its viscosity [19], solubility [20], phase behaviour [21] and crystallisation [22]. In water, PEO 
molecules adopt an expanded coil structure as water is considered a good solvent for PEO. These fully expanded coils have sufficient space to arrange into a crystaline network with the neigbouring chains to form spherulites when the polymer concentration increases. The solvent quality can be reduced by adding salts which disrupt the water structure, reducing the favourable interaction between monomers and water until the theta point is reached, where the molecule is somewhat condensed and described by the statistics of an ideal coil. On the addition of further salt, the polymer will precipitate out of solution as it undergoes a coil-globule transition; several works have studied the effectiveness of various salts [23, 24]. An interesting follow-up paper studied the change in polymer conformation using optical tweezers to show the elasticity of a single PEO molecule as the salt concentration is altered [25].

In a previous work [26] we observed for the first time that aqueous droplets of PEO follows a unique drying route. The liquid droplet is squeezed inwards by a constricting ring of crystallising polymer at the contact line which eventually lifts the remaining liquid from the surface, forming polymer pillars which may be taller than the original droplet. The polymer crystallisation is an essential part of this process and explains why this effect has not been seen in other, non-crystallising polymers. Further work [27] examined in detail the mechanisms controlling this behaviour, showing that the effects of droplet volume, contact angle, temperature and vapour pressure could be combined into a dimensionless Péclet number which correctly predicts whether a given droplet will form a pillar or not. The physical mechanism was later expanded to also include the effects of polymer concentration [28] and polymer molecular weight [29]. More recently very similar pillar-forming behaviour was observed in droplets of blood evaporating at pressures below $10 \%$ atmospheric pressure. The striking similarities between the two cases were used to develop a generalised classification of droplet evaporation modes [30], determined by the constant $A$ which relates the instantaneous droplet radius $R$ to the speed of motion of the contact line $\dot{R}$ in the equation $\dot{R}=-\frac{A}{R}$. For $A=0$, the contact line is pinned and evaporation is through CCR mode, resulting in classic ring-stain deposit. For $0<A<A_{\mathrm{CCA}}$, less than $A_{\mathrm{CCA}}$ a specific rate at which the droplet evaporates with constant contact angle, the deposit is a doughnut shape due to the slow receding of the contact line during evaporation. For $A>A_{\mathrm{CCA}}$, "fast receding" of the contact line is seen, resulting in the deposition of a tall pillar. In ref. [30] the parameter $A$ was varied using polymer concentration.

An aesthetically similar observation is seen in freezing water droplets. Liquid water expands as it solidifies, leading to cusped solid deposits [31]. Droplets of salt solutions do not typically form ring-stains due to modified wettability once salt crystals begin to precipitate [32], and can often be seen to "creep" across the surface, leaving a deposit that is larger than the original droplet $[33,34]$.

Others have also studied the drying behaviour of PEO droplets. Mamalis et al. [35] varied the molecular weight and substrate chemistry to highlight the role of interfacial friction on pillar formation. $\mathrm{Hu}$ et al. [36] placed $5 \%$ concentration droplets of molecular weight $M_{\mathrm{W}}=$ $280 \mathrm{~kg} \mathrm{~mol}^{-1}$ PEO on both isothermal and heated substrates and found evidence of Marangoni flow at higher temperatures. Choi et al. [37] added $1 \mu \mathrm{m}$ and $6 \mu \mathrm{m}$ hollow glass spheres to very dilute (maximum of $0.1 \%$ ) PEO solutions with $M_{\mathrm{W}}=200 \mathrm{~kg} \mathrm{~mol}^{-1}$ and $900 \mathrm{~kg} \mathrm{~mol}^{-1}$ in order to alter the viscous drag on the moving particles. They found that even at such low concentrations, the effect of the polymer was sufficient to disrupt formation of the ring-stain.

In this work we investigate two methods to control the pillar formation in aqueous PEO droplets, and propose a simple method to characterise the basic shape of the deposit. Firstly we study mixtures of PEO with polymer microparticles, mapping out how the droplet deposits change from ring-stain to pillars as the relative concentration of the two components are varied. We use two different sized particles and compare their effects, to determine whether polymer crystallisation and hence pillar formation is disrupted more by particle volume fraction, or number density. Secondly we disrupt the water structure by adding to the PEO solutions small quantities of the salt potassium sulphate, $\mathrm{K}_{2} \mathrm{SO}_{4}$, as this ranks relatively highly in both the Hoffmeister series (indicating a strong tendecy to induce protein precipitation) and in the data in ref. [23] on the effect of various salts on PEO solubility. With added salt the solution is not as good a solvent for the polymer, so its quality as a solvent has reduced. We study how the pillar formation is disrupted when the solvent quality is reduced leading to a reduction in the size of the dissolved polymer coil and a restriction on the freedom of the coils to form ordered crystalline structures. To quantify our findings from both sets of experiments, we present a novel and versatile technique, using the skewness of a height profile, to distinguish quantitatively between the various deposits.

\section{Methods}

Polymer solutions were prepared by dissolving PEO powder (from Sigma Aldrich with average molecular weight $M_{\mathrm{w}}=100 \mathrm{~kg} \mathrm{~mol}^{-1}$ and $200 \mathrm{~kg} \mathrm{~mol}^{-1}$, referred to as PEO100 and PEO200) in distilled, deionized water with a range of mass concentrations $c_{0}$ and left to equilibrate for at least 24 hours. The particle suspensions used in this study were surfactant-free polystyrene spheres commercially available from Sigma-Aldrich with particle diameters of $0.50 \pm 0.05 \mu \mathrm{m}$ and $5.0 \pm 0.5 \mu \mathrm{m}$, supplied at up to $10 \%$ solids by volume and a particle density of $1.050 \mathrm{~g} \mathrm{~cm}^{-3}$ at $20^{\circ} \mathrm{C}$. The suspensions were diluted with deionized water, if necessary, and added to the PEO solutions to obtain samples with particle concentrations by mass $c_{\mathrm{p}}$ between $1 \%$ and $5 \%$. Due to the difficulties of dissolving PEO into the particle solutions, the highest polymer concentrations possible were around $16 \%$. The salt $\mathrm{K}_{2} \mathrm{SO}_{4}$ was added to other PEO solutions to give salt concentrations by mass $c_{\mathrm{s}}$ between $0.1 \%$ and $1.0 \%$. As the salt comes in dry powdered form, it is possible to mix solutions 
with polymer concentrations as high as $25 \%$. All samples were left on a SRT6-Stuart roller mixer for 12 hours to minimise the agglomeration of particles. The mixing process was repeated for at least 30 minutes prior to every experiment.

For the evaporation experiments, droplets of initial volume $V_{0}$ between $0.4 \mu \mathrm{l}$ and $5 \mu \mathrm{l}$ were slowly pipetted onto clean glass slides using a positive displacement Gilson pipette to ensure accurate dispensing of the viscous solutions. Previous work [27] has shown that, provided the droplet dimensions are smaller than the capillary length (around $2 \mathrm{~mm}$ ), droplet volume does not affect deposition patterns. Samples were imaged from the side during drying using an Imaging Source CCD camera with IC Capture software, illuminated by a Stocker Yale diffuse back light (ML-045). ImageJ was used to analyse the images and extract the profile coordinates of the deposit from the images of the final deposit. Images of the final deposits were also taken using a Nikon Eclipse TE2000-S inverted microscope and an Olympus BX51 upright microscope using crossedpolarisers to highlight the crystallised polymer spherulites.

For the droplets containing polymer plus salt, ten droplets at each combination were deposited, and although not all were usable for analysis, in total 380 dried droplets were imaged and analysed. For the droplets of polymer plus particls, ten droplets were deposited at each composition, with profile images taken of three. As some of the samples formed ring-stains, in which the centre was lower than the edge, images taken from the side were not able to capture the profile accurately. In these situations, the height profile of the deposits was analysed using a Dektat 150 surface profiler with a $1 \mathrm{mg}$ scan force to reduce the chance of damage to the delicate samples. The profiler recorded a line profile for each deposit with around $3 \mu \mathrm{m}$ horizontal resolution and almost $1 \mathrm{~nm}$ vertical resolution. For each droplet, 6 scans were taken along diameters, spaced evenly around the deposit.

\section{Results}

Tables showing representative final images for the deposits, both profile and overhead, for both particle sizes and salt, are presented in figs. 1 to 6 . The axes of the table for the salt samples have been transposed owing to the large number of different concentrations used. Repeatability for all droplets was very good, as illustrated by the superimposed profiles presented in fig. 1. Although measurements were not taken from all droplets, the ten repeats for each combination were compared by eye and in nearly all cases the deposits were very similar, with slightly more variation seen with droplets at the boundary between flat and pillar structures.

Pillar formation, which is typically seen above $3 \%$ in pure PEO droplets, is shifted to higher concentrations with the addition of particles, with the $5 \mu \mathrm{m}$ particles in particular disrupting the pillars. Between $6 \%$ and $10 \%$ the pillars are more rounded and at their base extend to the full width of the initial droplet. For all samples, steep, tall pillars are seen at high polymer concentrations. As shown

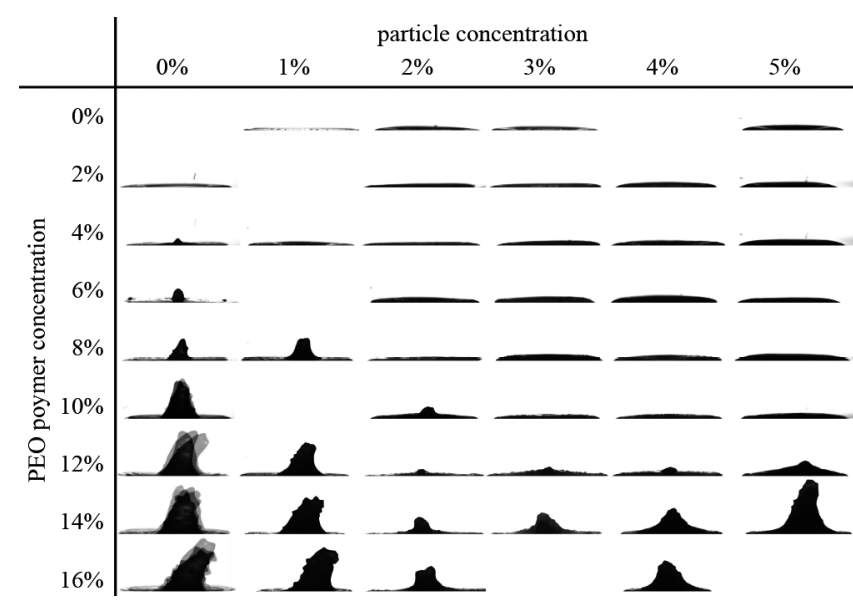

Fig. 1. Final profile images for $0.7 \mu \mathrm{l}$ droplets containing PEO100 and $5 \mu \mathrm{m}$ particles with $c_{0}$ between $0 \%$ and $16 \%$ and $c_{\mathrm{p}}$ between $0 \%$ and $5 \%$. The lower four pure polymer images show superimposed profiles from repeated droplets to indicate experimental repeatability.

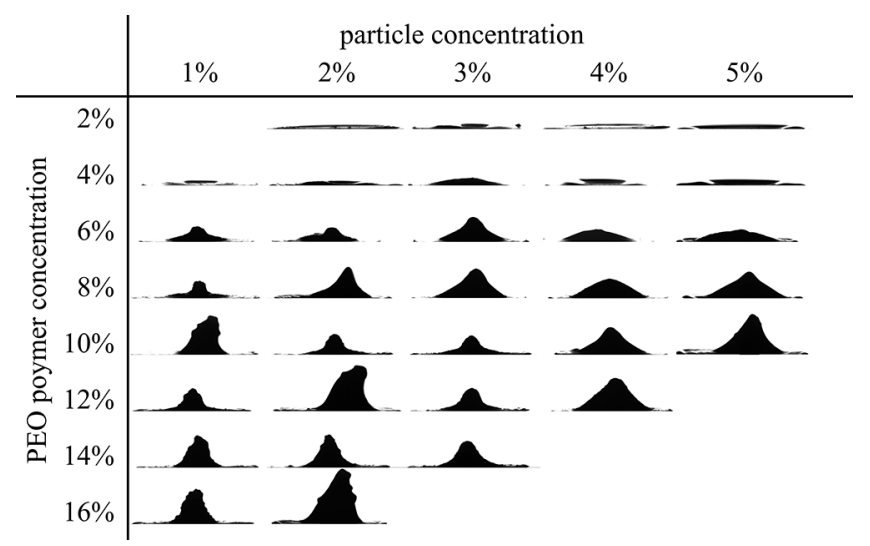

Fig. 2. Final profile images for $0.7 \mu \mathrm{l}$ droplets containing PEO100 and $0.5 \mu \mathrm{m}$ particles with $c_{0}$ between $2 \%$ and $16 \%$ and $c_{\mathrm{p}}$ between $1 \%$ and $5 \%$.

in fig. 2, the disruption is less pronounced for the $0.5 \mu \mathrm{m}$ particles.

The overhead images of the droplets with $5 \mu \mathrm{m}$ particles are uniformly dark and did not reveal any particular variations between droplets, so are not included here. However, higher magnification microscopy close to the contact line shows that for low polymer concentrations, particles were deposited at the edge in a ringstain, whereas at higher $c_{0}$, particles were more evenly distributed, as illustrated in fig. 4.

The results for samples with no polymer and shown in fig. 5. The particle and water droplets show classic ringstains while the salt and water solutions show the more complicated crystal rings seen in such "creeping" solutions where the liquid spreads out over the deposited solid [32, 33]. The particles deposits were also characterised using a stylus profilometer.

The total drying time did not vary significantly between the various droplets and was around 600 seconds at standard atmospheric conditions. 


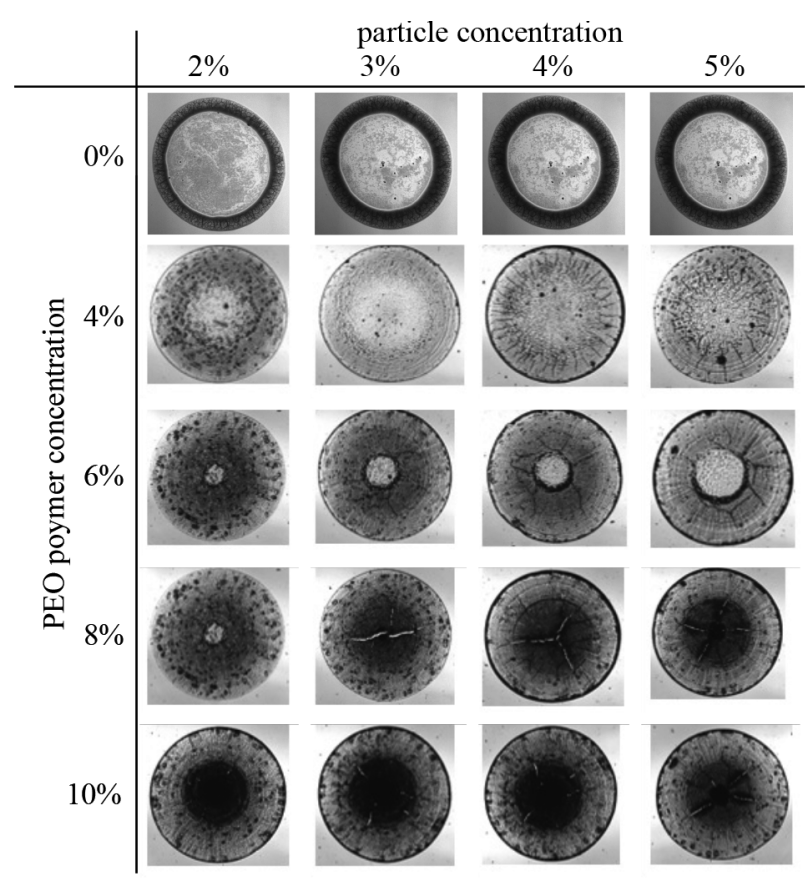

Fig. 3. Final overhead images taken through crossed polarisers of $0.4 \mu \mathrm{l}$ droplets containing PEO100 and $0.5 \mu \mathrm{m}$ particles with $c_{0}$ between $0 \%$ and $10 \%$ and $c_{\mathrm{p}}$ between $2 \%$ and $5 \%$. Ringstains are clear at low values of $c_{0}$ and at higher values (above around $6 \%$ ) pillars are present, seen as a central circle in the images.

The deposit shapes are more usefully quantified using surface profile data giving the height of the deposit $h(r)$ as a function of distance from the droplet centre $r$. As we have shown previously [3], robust ring-stains are observed over a range of experimental conditions, and here we confirm this result, finding clear ring-stains for all particle droplets without PEO, $c_{0}=0 \%$, as evidenced by the $c_{0}=0 \%$ curve in fig. 7 for $0.5 \mu \mathrm{m}$ particles. Note that both the height profiles and microscopy reveal that there is at most a monolayer of particles deposited in the centre of the ring. The other curves in this figure show the effect of increasing PEO concentration: at low values of $c_{0}$, we still observe a ring-stain however with non-zero height in the centre. Figure 4 suggests that there may be separation between particles and polymer, as the ring preferentially contains particles, and the centre contains polymer. For higher concentrations, $c_{0} \geq 6.0 \%$, a central pillar is clearly defined, which increases in height with polymer concentration. The measured profiles using $5 \mu \mathrm{m}$ particles are not presented, but show a similar transition from ring-stain to pillar on the addition of PEO.

\section{Discussion}

We have shown qualitatively in the previous section that the addition of either particles or salt can disrupt the formation of PEO polymer pillars. It has been established previously [26] that pillars are deposited following a four-
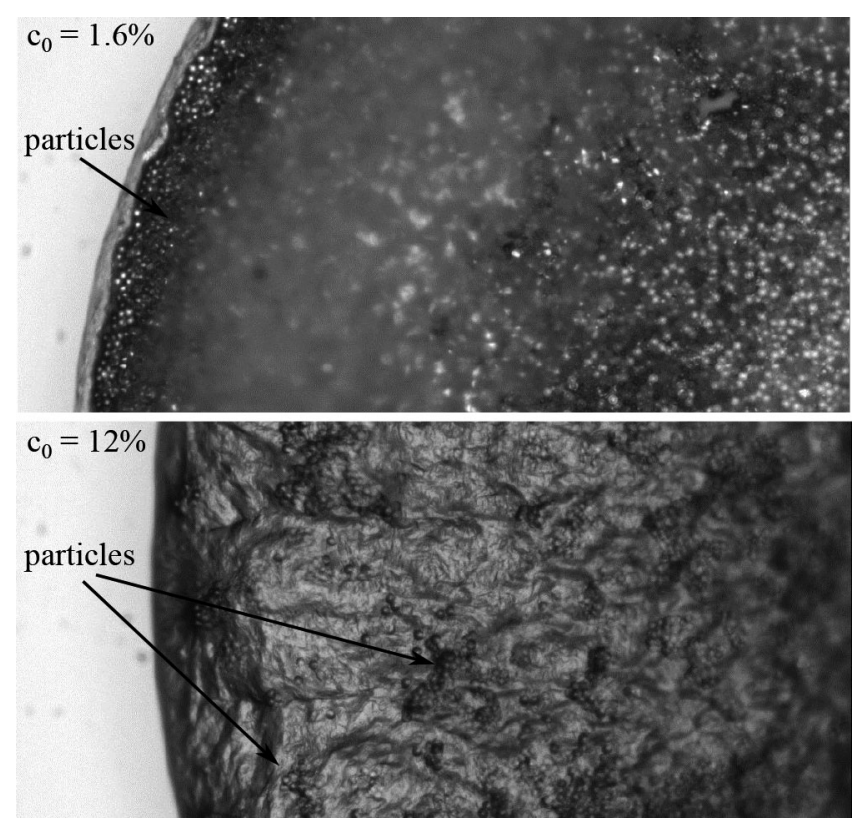

Fig. 4. Close-up images of the contact line of $0.4 \mu \mathrm{l}$ droplets with $c_{0}$ of $1.6 \%$ and $12 \%$ and concentration of $5 \mu \mathrm{m}$ particles $c_{\mathrm{p}}=4 \%$. At low $c_{0}$, particles are preferentially deposited at the edge, like a classic ring-stain despite the presence of polymer. At higher concentrations, the polymer disrupts the particle movement and consequently, particles are distributed more evenly.

stage process, where the critical step in the vertical growth of the structures is the "bootstrap" step in which crystalline polymer spherulites are deposited at the contact line and bind preferentially to the glass substrate creating a solid collar around the reminaing liquid. Due to additional loss of water, the collar contracts, squeezing the droplet towards the centre. As the droplet recedes, the collar begins to squeeze the liquid upwards, away from the substrate, which is particularly dramatic at low pressures [27]. When the crystallisation process is hindered, the collar will not form, the contact line will not recede and pillars will not be able to form [29]. The addition of $\mathrm{K}_{2} \mathrm{SO}_{4}$ disrupts the structure of PEO in aqueous solutions and is known to initiate precipitation of the polymer, by decreasing the affinity of the polymer monomers for water compared to the polymer-polymer attractions. The polymer chains collapse from their open, expanded coil state, in which they are described by the statistics of a self-avoiding chain to a more compact ideal random walk before preciptiating out of solution in the very compact globule state [25]. This configurational change of the polymer should prevent pillar formation at low salt concentrations, well below $1 \%$. Although not of primary focus in this work, a very low concentration of polymer $\left(c_{\mathrm{p}}<2 \%\right)$ was observed to disrupt the creeping behaviour of pure salt droplets.

In the polymer plus particle system, the polymer has a dramatic effect on the formation of the ring-stain. The overall deposit becomes flat at low polymer concentrations, although there does appear to be some evidence for 


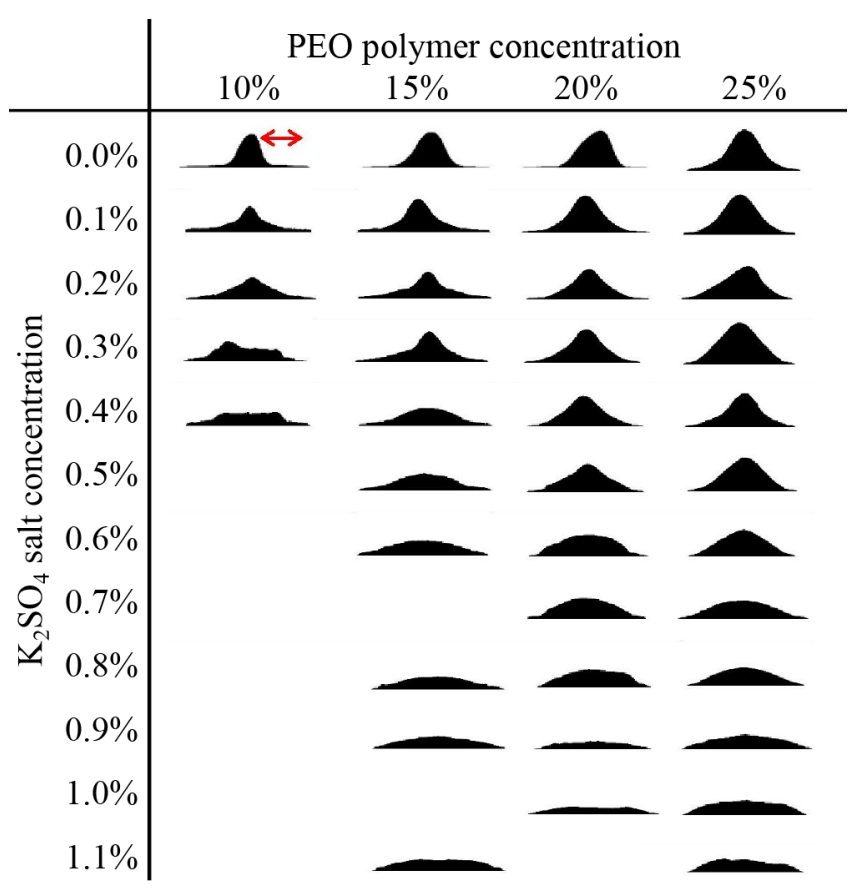

Fig. 5. Final profile images for $5 \mu \mathrm{l}$ droplets containing PEO200 and $\mathrm{K}_{2} \mathrm{SO}_{4}$ with $c_{0}$ between $10 \%$ and $25 \%$ and $c_{\mathrm{s}}$ between $0 \%$ and $1.1 \%$.

fractionation within the droplet, with particles preferentially depostied at the edge for $c_{\mathrm{p}}<2 \%$. The effect discussed above, in which polymer crystallisation at the contact line drives the droplet inwards, is sufficiently strong to overcome the self-pinning that occurs typically at the edge of evaporating suspension droplets, preventing the ring-stain. The effect of the particles on pillar formation is less dramatic than the effet of salt, with the $5 \mu \mathrm{m}$ particles shifting the threshold concentration for pillars up from $3 \%$ to around $12 \%$, and the smaller $0.5 \mu \mathrm{m}$ particles making very little difference to the threshold values of $c_{\mathrm{p}}$. This suggests that pillar formation is relatively immune to physical impurities, which only provide a steric rather than a configurational hindrance to polymer crystallisation. Even the smallest particles are significantly larger than the polymer molecules themselves, so do not interfere with the molecular process of crystallisation. There may in fact be an optimum concentration of particles around $3 \%$ to disrupt pillar formation, as shown in fig. 1, as at $5 \%$ the pillars seem to be becoming larger again.

In order to quantify how the shape of the deposits are altered we chose to measure the skewness of the droplet profile, and in keeping with statistical textbooks assign the variable $\gamma_{1}$. Skewness, which is related to the third moment of a distribution (as mean is related to the first moment and standard deviation to the second moment), indicates whether a distribution function leans to the left or right. As illustrated in fig. 7, this parameter distinguishes the three main types of pillar deposit: profiles which are peaked towards $r=0$ have a positive skewness; ring-stain deposits in which the profile is peaked towards the edge at $r=R$ have a corresponding negative

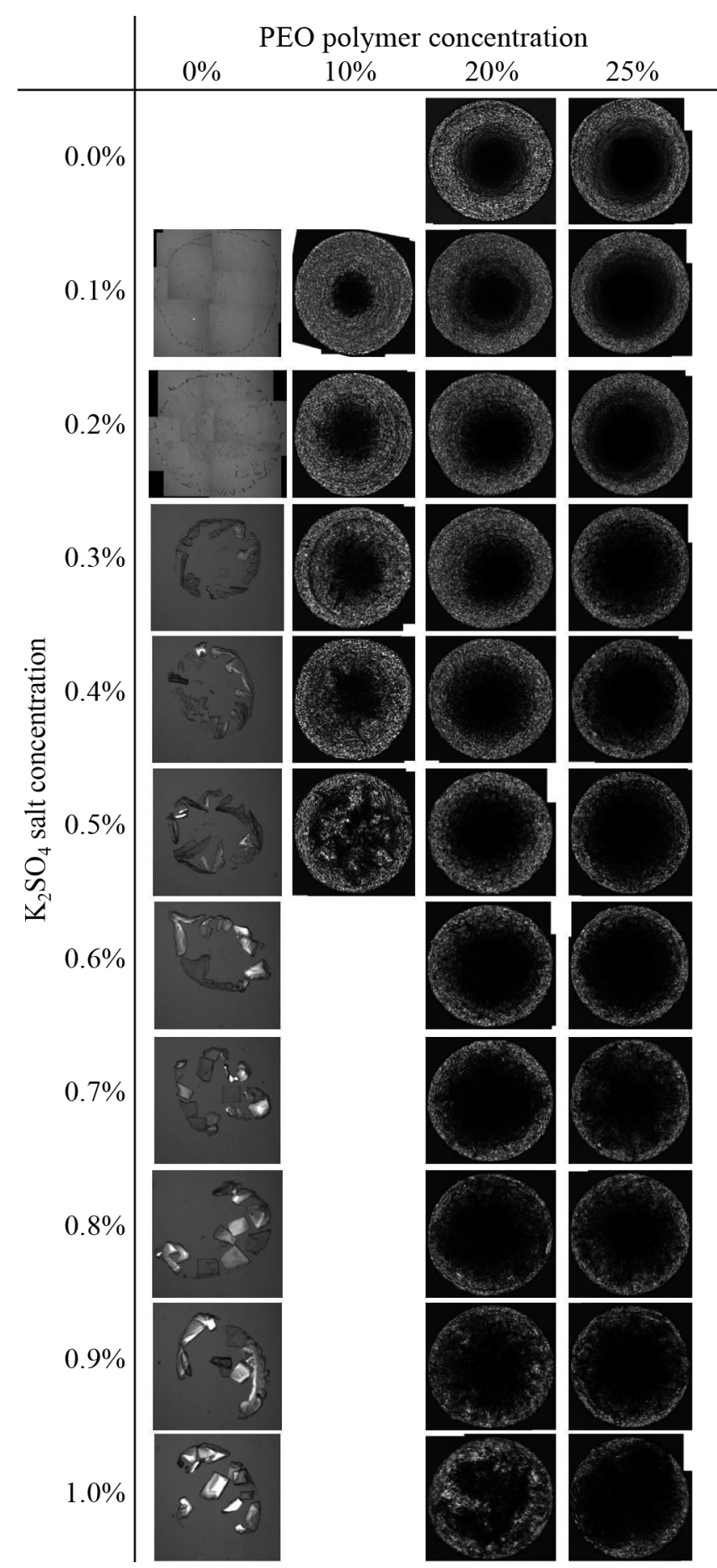

Fig. 6. Final overhead images taken through crossed polarisers for $5 \mu \mathrm{l}$ droplets containing $\mathrm{PEO} 200$ and $\mathrm{K}_{2} \mathrm{SO}_{4}$ with $c_{0}$ between $0 \%$ and $25 \%$ and $c_{\mathrm{s}}$ between $0 \%$ and $1.0 \%$.

skew; and symmetrical flat deposits have a skewness close to zero. The simpler approach of using the value of $r$ at which the droplet height $h(r)$ is a maximum does not take into account the height of the peak, and therefore fails to consistently identify flattish deposits, where the maximum is not clearly defined. The use of skewness overcomes this problem. 


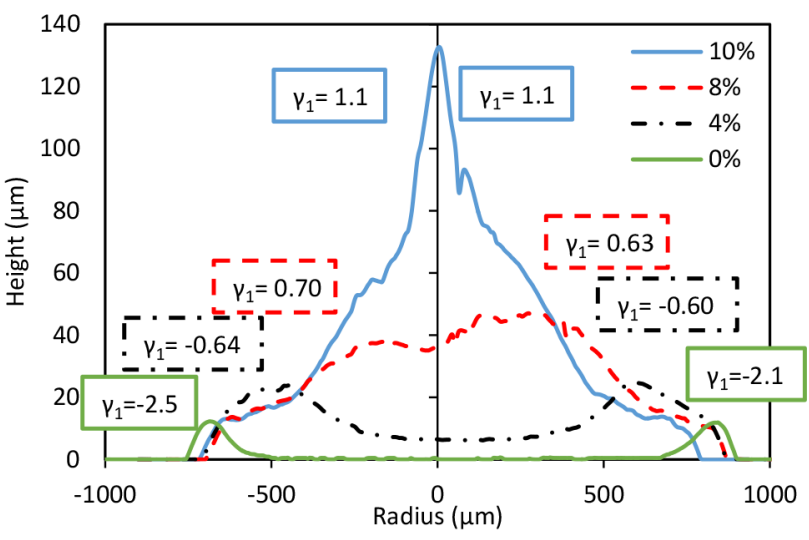

Fig. 7. Deposit profiles for $0.4 \mu \mathrm{l}$ droplets with polymer concentration $c_{0}$ between $0 \%$ and $10 \%$ and concentration of $0.5 \mu \mathrm{m}$ particles fixed at $c_{\mathrm{p}}=4 \%$. The values of the skewness $\gamma_{1}$ for both left and right sides of each profile are also indicated on the figure. With no polymer, $c_{0}=0 \%$, the profile of a classic ringstain is seen, with negative skewness $\gamma_{1}<-2$. For $c_{0}=4 \%$ a ring-like deposit at the edge is seen, with additional deposit in the centre and $\gamma_{1} \approx-0.6$. For $c_{0}=8 \%$, the deposit is a short pillar with $\gamma_{1} \approx 0.66$ and for $c_{0}=10 \%$ a tall pillar is seen with $\gamma=1.1$.

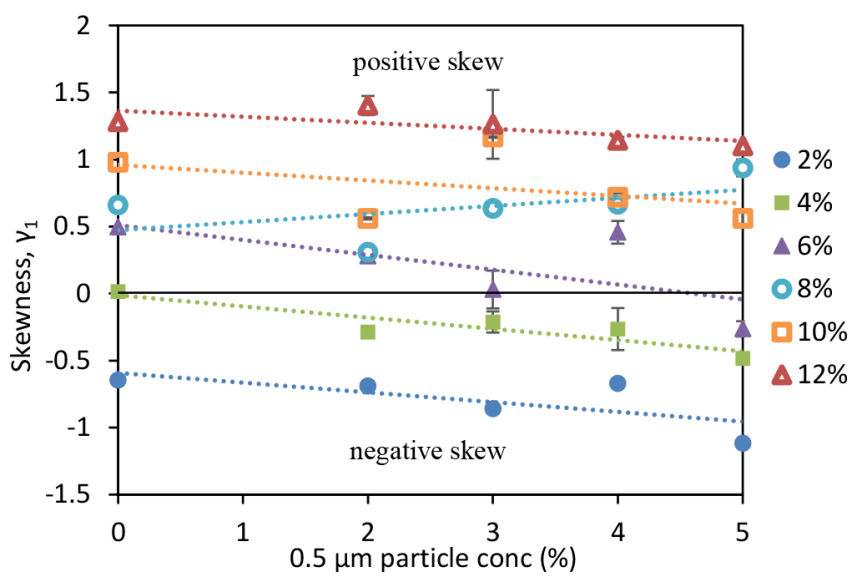

Fig. 8. Skewness of final deposit as a function of added concentration of $0.5 \mu \mathrm{m}$ particles into $0.7 \mu \mathrm{l}$ droplets containing PEO100 at various concentrations. Vertical error bars indicate the difference in skew between the two sides, average over three droplets. Uncertainties in PEO concentration are around $0.5 \%$.

The simplest definition of skew is the non-parametric skew, defined as the difference between the median and the mean $\bar{r}$ of the distribution, divided by the standard deviation. However, this approach did not reliably capture the shape of the distributions, so for our analysis we used the standardised third moment of the radial profile. As the data obtained from both image analysis of the interface and surface profilometery is discrete with values of $r_{i}, h_{i}$ (and an increment $\delta_{i}=r_{i+1}-r_{i}$ to account for data that are not necessarily equally spaced), we used the following formulae to calculate the skew:

$$
\gamma_{1}=\frac{\sum\left(r_{i}-\bar{r}\right)^{3} h_{i} \delta_{i}}{\left[\sum\left(r_{i}-\bar{r}\right)^{2} h_{i} \delta_{i}\right]^{3 / 2}} .
$$

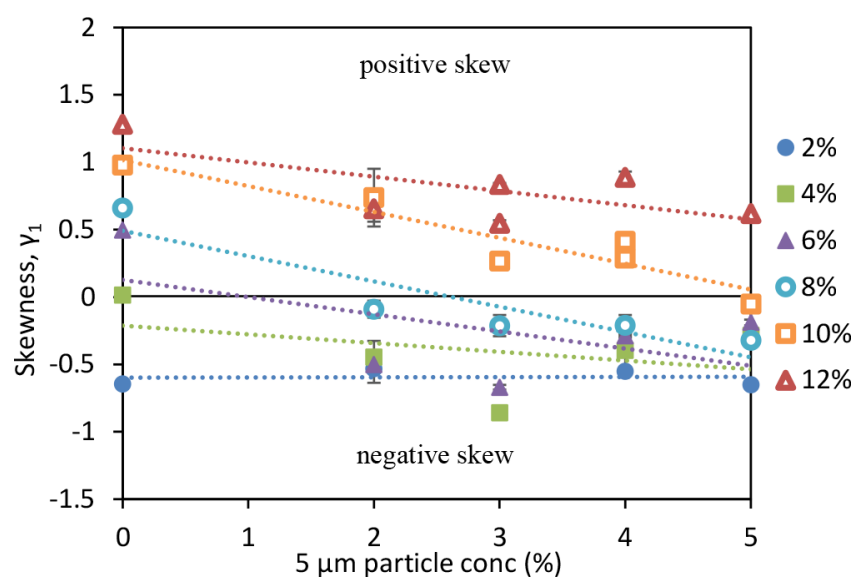

Fig. 9. Skewness of final deposit as a function of added concentration of $5 \mu \mathrm{m}$ particles into $0.7 \mu \mathrm{l}$ droplets containing PEO100 at various concentrations. Vertical error bars indicate the difference in skew between the two sides, averaged over three droplets. Uncertainties in PEO concentration are around $0.5 \%$.

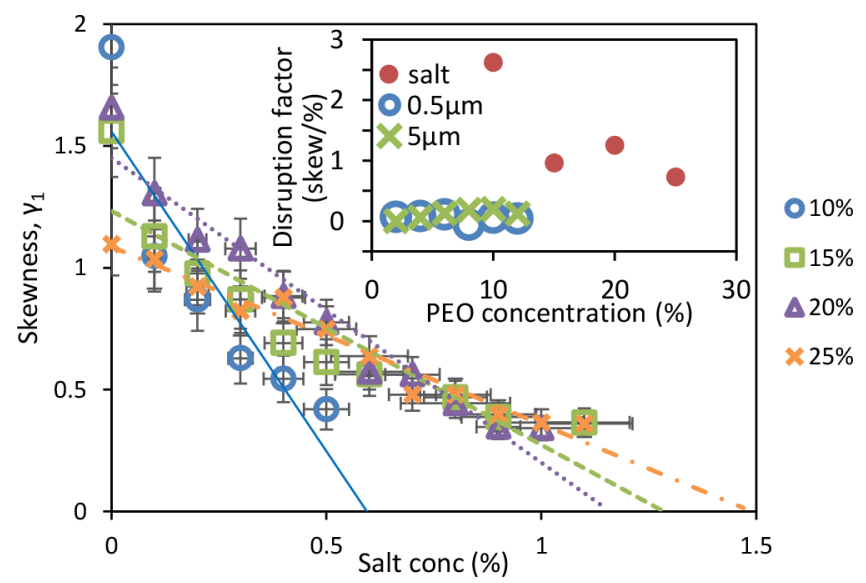

Fig. 10. Skewness of final deposit as a function of added concentration of $\mathrm{K}_{2} \mathrm{SO}_{4}$ salt to droplets containing PEO200 at various concentrations. Vertical error bars indicate the standard deviation of skewness measured over 8 droplets. Uncertainties in PEO concentration are around $0.5 \%$. The inset shows the disruption factor for each of the three systems as a function of PEO concentration, showing salt to have a larger effet than either size of particle.

For a given distribution, with $r$ ranging from $-R$ to $+R$ we calculated the average of $\gamma_{1}$ for $0<r<R$ for the right-hand side and $-\gamma_{1}$ for $-R<r<0$ for the left hand side, the difference between the two halves characterising the uncertainty in the skewness.

The results for the skew analysis for all three systems are shown in figs. 8-10. Nearly all data sets show that the skewness of the deposit reduces with increasing additives, indicating that pillars become flatter. To quantify the effect of the additives, to a first approximation we fit the data for each value of $c_{0}$ with a straight line, acknowledging that a linear dependency does not necessarily give the best agreement with the data, particularly for the salt, and that in one or two cases the skew may even increase 
at higher concentrations. We recognise that there are limitations with this simple charactersiation of the shape of the droplet deposit, which for example does not capture the observed segregation between particle sand polymer, as this doesn not affect the overall height of the deposit.

We calculate a disruption factor to quantify this effect, equal to the negative of the slope of the best-fit lines. These are plotted in the inset to fig. 10 and emphasise that only a small quantity of salt is required to prevent pillar formation, with an average disruption factor around 1.4 skew $/ \%$. The disruption factors for the polymer plus particle systems are significantly less, 0.05 for the $0.5 \mu \mathrm{m}$ particles and 0.12 for the $5 \mu \mathrm{m}$ particles, with the smaller particles having less of an effect on the pillar formation process in agreement with the qualitative observations made from the droplet profiles.

\section{Conclusions}

We have investigated the effect of adding two different sizes of micro-particles to evaporating droplets of poly(ethylene oxide) solution. In pure solutions, PEO forms tall pillars above around $3 \%$ concentration and micro-particles typically leave ring-stain deposits. We have shown particles disrupt pillar formation and polymer disrupts ring-stain formation. Pillar formation is shifted to slightly higher polymer concentrations when large $5 \mu \mathrm{m}$ particles are added, but is less affected by the addition of $0.5 \mu \mathrm{m}$ particles. We also show preliminary evidence for particle segregation at higher polymer concentrations. This observation opens up the possibility of using PEO pillars to create functional pillars containing bespoke particles, with the confidence that the additional impurities, particularly small particles, are not likely to interfere with pillar formation. Potassium sulfate is known to reduce the affinity of PEO with water, causing the coils to collapse. By adding this salt to evaporating PEO droplets we see significant interference of the pillar formation. To quantify the different deposits observed we introduce the use of the normalised third moment of the deposit profile, commonly known as the skewness. We show that this simple parameter seems to capture the three deposit types, with a positive skewness for pillars, close to zero for flat deposits and negative skewness for ring-stains. The skew values can also be used to define a disruption factor which quantifies how effect different additives are at changed the shape of the deposit. We believe skewness has the potential to be very useful for characterisation of deposits in a wide variety of systems.

Y. Msambwa is funded by the Tanzanian government through the Dar Es Salaam University College of Education. D.J. Fairhurst would like to thank NTU for funding a research sabbatical. We also acknowledge useful discussions initiated through COST Action MP1106. We are very grateful to the referees for making several insightful comments.
Open Access This is an open access article distributed under the terms of the Creative Commons Attribution License (http://creativecommons.org/licenses/by/4.0), which permits unrestricted use, distribution, and reproduction in any medium, provided the original work is properly cited.

\section{References}

1. R.D. Deegan, O. Bakajin, T.F. Dupont, G. Huber, S.R. Nagel, T.A. Witten, Nature 389, 827 (1997).

2. R.G. Picknett, R. Bexon, J. Colloids Interface Sci. 61, 336 (1977).

3. Yohana Msambwa, David J. Fairhurst, Fouzia Ouali, Interfacial Phenom. Heat Transf. 1, 207 (2013).

4. Peter J. Yunker, Tim Still, Matthew A. Lohr, A.G. Yodh, Nature 476, 308 (2011).

5. B.M. Weon, J.H. Je, Phys. Rev. E 82, 015305 (2010).

6. H. Hu, R.G. Larson, J. Phys. Chem. B 106, 1334 (2002).

7. H.B. Eral, D. Mampallil Augustine, M.H.G. Duits, F. Mugele, Soft Matter 7, 4954 (2011).

8. X. Shen, C.M. Ho, T.S. Wong, J. Phys. Chem. B 114, 5269 (2010).

9. Yanshen Li, Cunjing Lv, Zhaohan Li, David Quéré, Quanshui Zheng, Soft Matter 11, 4669 (2015).

10. Khellil Sefiane, Adv. Colloid Interface Sci. 206, 372 (2014).

11. L. Pauchard, C. Allain, Europhys. Lett. 62, 897 (2003).

12. M.A. Rodríguez-Valverde, P. Ramón-Torregrosa, A. PáezDueñas, M.A. Cabrerizo-Vílchez, R. Hidalgo-Álvarez, Adv. Colloid Interface Sci. 136, 93 (2008).

13. L. Wallstronm, K.A.H. Lindberg, Wood Sci. Technol. 29, 109 (1995).

14. A.A. Collyer, Phys. Educ. 10, 305 (1975).

15. Andre C. Dumetz, Rachael A. Lewus, Abraham M. Lenhoff, Eric W. Kaler, Langmuir 24, 10345 (2008).

16. A. Abuchowski, T. Vanes, N.C. Palczuk, F.F. Davis, J. Biol. Chem. 252, 3578 (1977).

17. Boualem Hammouda, J. Polym. Sci. Part B - Polym. Phys. 44, 3195 (2006).

18. B. Hammouda, D.L. Ho, S. Kline, Macromolecules 37, 6932 (2004).

19. M. Mohsen-Nia, H. Modarress, H. Rasa, J. Chem. Engin. Data 50, 1662 (2005).

20. Stefan Bekiranov, Robijn Bruinsma, Philip Pincus, Phys. Rev. E 55, 577 (1997).

21. Ana Saraiva, Ole Persson, Aage Fredenslund, Fluid Phase Equilib. 91, 291 (1993).

22. Ni Ding, Eric J. Amis, Macromolecules 24, 3906 (1991).

23. F.E. Bailey Jr., R.W. Callard, J. Appl. Polym. Sci. 1, 56 (1959).

24. Ebba Florin, Roland Kjellander, Jan Christer Eriksson, J. Chem. Soc. Faraday Trans. 1: Phys. Chem. Condens. Phases 80, 2889 (1984).

25. A. Dittmore, D.B. McIntosh, S. Halliday, O.A. Saleh, Phys. Rev. Lett. 107, 148301 (2011).

26. David Willmer, Kyle Anthony Baldwin, Charles Kwartnik, David John Fairhurst, Phys. Chem. Chem. Phys. 12, 3998 (2010).

27. Kyle Anthony Baldwin, Manon Granjard, David I. Willmer, Khellil Sefiane, David John Fairhurst, Soft Matter 7, 7819 (2011). 
28. Kyle A. Baldwin, Samuel Roest, David J. Fairhurst, Khellil Sefiane, Martin E.R. Shanahan, J. Fluid Mech. 695, 321 (2012).

29. K.A. Baldwin, D.J. Fairhurst, Colloid. Surf. A 441, 867 (2014).

30. Kyle Anthony Baldwin, David John Fairhurst, Soft Matter 11, 1628 (2015).

31. Alvaro G. Marin, Oscar R. Enriquez, Philippe Brunet, Pierre Colinet, Jacco H. Snoeijer, Phys. Rev. Lett. 113 054301 (2014).

32. D.J. Fairhurst, Droplets of Ionic Solutions, chapt. 20 in Droplet wetting and evaporation: from pure to complex flu$i d s$, edited by David Brutin (Elsevier, 2015) pp. 295-314.
33. Willem J.P. van Enckevort, Jan H. Los, Crystal Growth Design 13, 1838 (2013).

34. Noushine Shahidzadeh, Marthe F.L. Schut, Julie Desarnaud, Marc Prat, Daniel Bonn, Sci. Rep. 5, 5908 (2015).

35. Dimitrios Mamalis, Vasileios Koutsos, Khellil Sefiane, Antonia Kagkoura, Michail Kalloudis, Martin E.R. Shanahan, Langmuir 428, 39 (2015).

36. Yin-Chun $\mathrm{Hu}$, Qiong Zhou, Hai-Mu Ye, Yu-Feng Wang, LiShan Cui, Colloids Surf. A: Physicochem. Engin. Aspects 428, 39 (2013).

37. Yongjoon Choi, Jeongin Han, Chongyoup Kim, Korean J. Chem. Engin. 28, 2130 (2011). 